\title{
Efeitos da música na ansiedade de doadores de sangue: ensaio clínico randomizado
}

Effects of music on the anxiety of blood donors: randomized clinical trial

Efectos de la música en la ansiedad de donantes de sangre: ensayo clínico aleatorizado

Karla Fabiana Nunes da Silva ${ }^{1}$ iD https://orcid.org/0000-0003-4140-7408

Márcia Marques dos Santos Felix ${ }^{1}$ io https://orcid.org/0000-0001-8431-6712

Luciana Falcão da Cruz ${ }^{1}$ io https://orcid.org/0000-0001-5903-3577

Elizabeth Barichello ${ }^{1}$ id https://orcid.org/0000-0001-7764-032X

Patrícia da Silva Pires ${ }^{1}$ id https://orcid.org/0000-0002-2537-3909

Ana Lúcia De Mattia ${ }^{1}$ id https://orcid.org/0000-0001-7912-4943

Vanderlei José Haas ${ }^{1}$ io https://orcid.org/0000-0001-8743-0123

Maria Helena Barbosa ${ }^{1}$ id https://orcid.org/0000-0003-2749-2802

Como citar:

Silva KF, Felix MM, Cruz LF, Barichello E, Pires PS, Mattia AL, et al. Efeitos da música na ansiedade de doadores de sangue: ensaio clínico randomizado. Acta Paul Enferm. 2021;34:AAPE00461.

DOI

http://dx.doi.org/10.37689/actaape/2021A000461

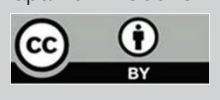

Descritores

Música; Ansiedade; Doadores de sangue; Sinais vitais; Hidrocortisona

Keywords

Music; Anxiety; Blood donors; Vital signs;

Hydrocortisone

Descriptores

Musica; Ansiedad; Donantes de sangre; Signos vitales; Hidrocortisona

Submetido

9 de Março de 2020

Aceito

1 de Junho de 2020

Autor correspondente

Maria Helena Barbosa

E-mail: mhelena331@hotmail.com / maria.barbosa@uftm.edu.br

\section{Resumo}

Objetivo: Avaliar os efeitos da música sobre a ansiedade-estado, parâmetros fisiológicos e laboratoriais, em doadores de sangue.

Métodos: Ensaio clínico randomizado, duplo-cego, realizado em um Hemocentro Regional, localizado no interior de Minas Gerais. Participaram do estudo 126 doadores de sangue, divididos aleatoriamente em dois grupos, sendo grupo experimental (intervenção musical antes da doação de sangue) e grupo controle (rotina padrão). Utilizou-se para a avaliação dos escores de ansiedade-estado, o Inventário de Ansiedade Traço-Estado (IDATE). A intervenção musical constitui-se de um repertório de músicas eruditas aplicadas através de fones de ouvidos, por aproximadamente 26 minutos. Para as variáveis quantitativas empregou-se análises descritivas, para análise das diferenças entre os escores de ansiedade-estado, frequência cardíaca e respiratória, utilizou-se Teste t Student e, Teste não paramétrico de Mann-Whitney para avaliar a diferença entre os valores de pressão arterial, saturação de oxigênio e níveis de cortisol.

Resultados: 0 grupo submetido à intervenção musical não apresentou redução estatisticamente significativa dos escores de ansiedade-estado ( $p=0,31)$. Entretanto, observou-se reduções significativas na frequência cardíaca $(p=0,006)$, frequência respiratória $(p=0,007)$ e níveis de cortisol sanguíneo $(p<0,001)$.

Conclusão: A música não reduziu os níveis de ansiedade-estado. Contudo, foi possível demonstrar a eficácia da intervenção na redução de parâmetros fisiológicos e laboratoriais, os quais apresentam-se alterados frente a situações ansiogênicas.

\section{Abstract}

Objective: To evaluate the effects of music on state-anxiety, physiological and laboratory parameters in blood donors.

Methods: Randomized, double-blinded clinical trial, conducted in a regional blood bank, located in the interior of the state of Minas Gerais, Brazil. In total, 126 blood donors participated in the study, randomly divided into two groups, being one experimental group (musical intervention before blood donation) and one control group (standard routine). To assess the state-anxiety scores, the State-Trait Anxiety Inventory (STAl) was used. The musical intervention consists of a repertoire of classical songs played through headphones, lasting approximately 26 minutes. For the quantitative variables, descriptive analyses were used to analyze the differences between state-anxiety, heart rate and respiratory rate, Student's t- test and Mann-Whitney's nonparametric test to evaluate the difference between blood pressure, oxygen saturation and cortisol levels.

Results: The group submitted to musical intervention did not present a statistically significant reduction in state-anxiety scores $(p=0.31)$. Nevertheless, significant reductions in heart rate $(p=0.006)$, respiratory rate

'Universidade Federal do Triângulo Mineiro, Uberaba, MG, Brasil.

Conflitos de interesse: "artigo original extraído da tese de doutorado - 0 efeito da música sobre a ansiedade de doadores de sangue: ensaio clínico randomizado apresentada ao Programa de Pós-Graduação em Atenção à Saúde da Universidade Federal do Triângulo Mineiro (UFTM), Uberaba, Minas Gerais, Brasil, em 2018. 
$(p=0.007)$ and blood cortisol levels $(p<0.001)$ were observed.

Conclusion: Music did not reduce the state-anxiety levels. We were able to demonstrate the effectiveness of the intervention in reducing physiological and laboratory parameters though, which are altered in the face of anxiogenic situations.

Registro Brasileiro de Ensaios Clínicos (ReBEC): RBR-6tvbdn

\section{Resumen}

Objetivo: Analizar los efectos de la música sobre la ansiedad-estado, parámetros fisiológicos y de laboratorio en donantes de sangre.

Métodos: Ensayo clínico aleatorizado, doble ciego, realizado en un centro de donación de sangre regional, ubicado en el interior del estado de Minas Gerais. Participaron en el estudio 126 donantes de sangre, divididos aleatoriamente en dos grupos: un grupo experimental (intervención musical antes de la donación de sangre) y un grupo de control (rutina normal). Para analizar la puntuación de la ansiedad-estado, se utilizó el Cuestionario de Ansiedad Estado Rasgo (IDATE). La intervención musical estaba compuesta por un repertorio de música erudita aplicada con auriculares, durante 26 minutos aproximadamente. Para las variables cuantitativas, se emplearon análisis descriptivos. Se utilizó el test-T Studentpara analizar las diferencias entre la puntuación de la ansiedad-estado, la frecuencia cardíaca y respiratoria y la prueba no paramétrica de Mann-Whitney para analizar la diferencia entre los valores de presión arterial, saturación de oxígeno y niveles de cortisol.

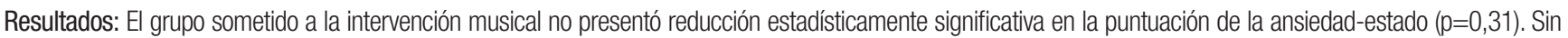
embargo, se observaron reducciones significativas en la frecuencia cardíaca $(p=0,006)$, frecuencia respiratoria $(p=0,007)$ y niveles de cortisol sanguíneo $(p<0,001)$.

Conclusión: La música no redujo los niveles de ansiedad-estado. No obstante, fue posible demostrar la eficacia de la intervención para la reducción de parámetros fisiológicos y de laboratorio, que se presentan alterados ante situaciones ansiógenas.

\section{Introdução}

A ansiedade é considerada um dos principais fatores que podem impactar negativamente no processo de doação de sangue, sobretudo no retorno de doadores, estando correlacionada, inclusive, à ocorrência de eventos adversos. ${ }^{(1)} \mathrm{A}$ ansiedade antes da doação de sangue, foi relacionada com sintomas de reaçóes vasovagais e considerada influência negativa na probabilidade de retorno de doadores, especialmente os doadores jovens, do sexo feminino e de primeira vez. ${ }^{(2)}$

Nos últimos anos, os resultados de vários estudos demonstraram a efetividade da música no controle e redução da ansiedade, em contextos variados. Pesquisas evidenciaram redução dos escores de ansiedade em pacientes cirúrgicos, oncológicos, em pacientes com insuficiência renal submetidos à hemodiálise, frente à realização de procedimentos invasivos e exames diagnósticos. ${ }^{(3-9)}$

Há indícios seguros de que a música, especialmente a relaxante, produz efeitos ansiolíticos por promover controle da apreensão e da ativação do sistema nervoso autônomo. ${ }^{(10)}$ Estudos evidenciaram que a música é capaz de baixar níveis elevados de estresse e que certos tipos de música, tais como a música meditativa ou clássica lenta, reduzem os marcadores neuro-hormonais de estresse e, consequentemente, indicadores fisiológicos como pressão arterial, frequência respiratória e cardíaca. ${ }^{(11,12)}$

A terapia musical é tida como uma intervenção autônoma do enfermeiro, que se centra, de acordo com o sistema de Classificação das Intervençóes de Enfermagem - Nursing Interventions Classification (NIC) —, no "uso da música para ajudar a alcançar uma mudança específica de comportamento, sentimento ou fisiologia”. ${ }^{(13)}$

De fato, estudos destacam que a utilização da música como tecnologia leve tem sido benéfica no contexto da enfermagem, sendo considerada, portanto, como intervenção não farmacológica simples, segura, de baixo custo e efetiva, que pode ser utilizada pela equipe de enfermagem como ferramenta complementar no cuidado. ${ }^{(14)}$

Frente ao exposto, este estudo teve como objetivo avaliar os efeitos da música sobre a ansiedade-estado, os parâmetros fisiológicos e laboratoriais, em doadores de sangue.

\section{Métodos}

Trata-se de um ensaio clínico randomizado (ECR), duplo-cego, prospectivo, realizado em um hemocentro localizado no interior de Minas Gerais, entre os meses de fevereiro e maio de 2018. Pesquisa conduzida segundo as recomendaçôes do Consolidated Standards of Reporting Trials (CONSORT) para ensaios que avaliam tratamentos não farmacológicos, ${ }^{(15,16)}$ registrada na plataforma de Registro Brasileiro de Ensaios Clínicos (REBEC), com o identificador primário, RBR-6tvbdn.

1. Participaram do estudo doadores de sangue de repetição, doadores esporádicos e de primeira 
vez, que efetuaram sua doação no hemocentro, durante o período de coleta de dados. $\mathrm{O}$ tamanho da amostra foi calculado para ambos os grupos, assumindo um nível de significância $\alpha=0,01$ e poder estatístico de $90 \%$, considerando os resultados obtidos em um estudo piloto, o qual evidenciou redução média no escore de ansiedade-estado de 1,60 $( \pm 1,14)$ para o grupo experimental e uma redução média de 0,60 $( \pm 1,67)$ para o grupo controle. Assim, obteve-se um tamanho amostral de 126 doadores, sendo 63 doadores no grupo experimental (GE) e 63 no grupo controle (GC).

2. Adotou-se como critérios de inclusão: candidatos à doação de sangue com idade igual ou superior a 18 anos, de ambos os sexos, aptos na triagem clínica e hematológica e que não estivessem em uso de medicamentos ansiolíticos. Excluiu-se os candidatos à doação com perda ou comprometimento auditivo autorreferido.

3. Foram selecionados para o estudo 126 candidatos à doação de sangue, dentre 1.089 can- didatos elegíveis, conforme demonstrado na figura 1.

$\mathrm{O}$ esquema de randomização utilizado neste estudo foi a randomização simples, realizado com auxílio de um site da web [http://www.randomization.com] que presta serviço de randomização online e gratuito. ${ }^{(17)}$ Esse processo foi realizado por um estatístico sem envolvimento clínico na pesquisa. Posteriormente à geração da sequência aleatória, gerou-se uma lista numerada sequencialmente para alocação dos doadores aos grupos. Ressalta-se que para garantir a ocultação da randomização dos participantes, a sua designação só foi de conhecimento do pesquisador responsável pela aplicação da intervenção, após entrar em contato com estatístico, o qual ficou com a lista e com a responsabilidade de fornecer tal designação quando solicitado.

Houve mascaramento do pesquisador que aplicou o instrumento de coleta de dados e dos técnicos do laboratório que realizaram a dosagem do cortisol quanto ao tipo de intervenção que cada participante recebeu, o que caracteriza este estudo como duplo-cego.

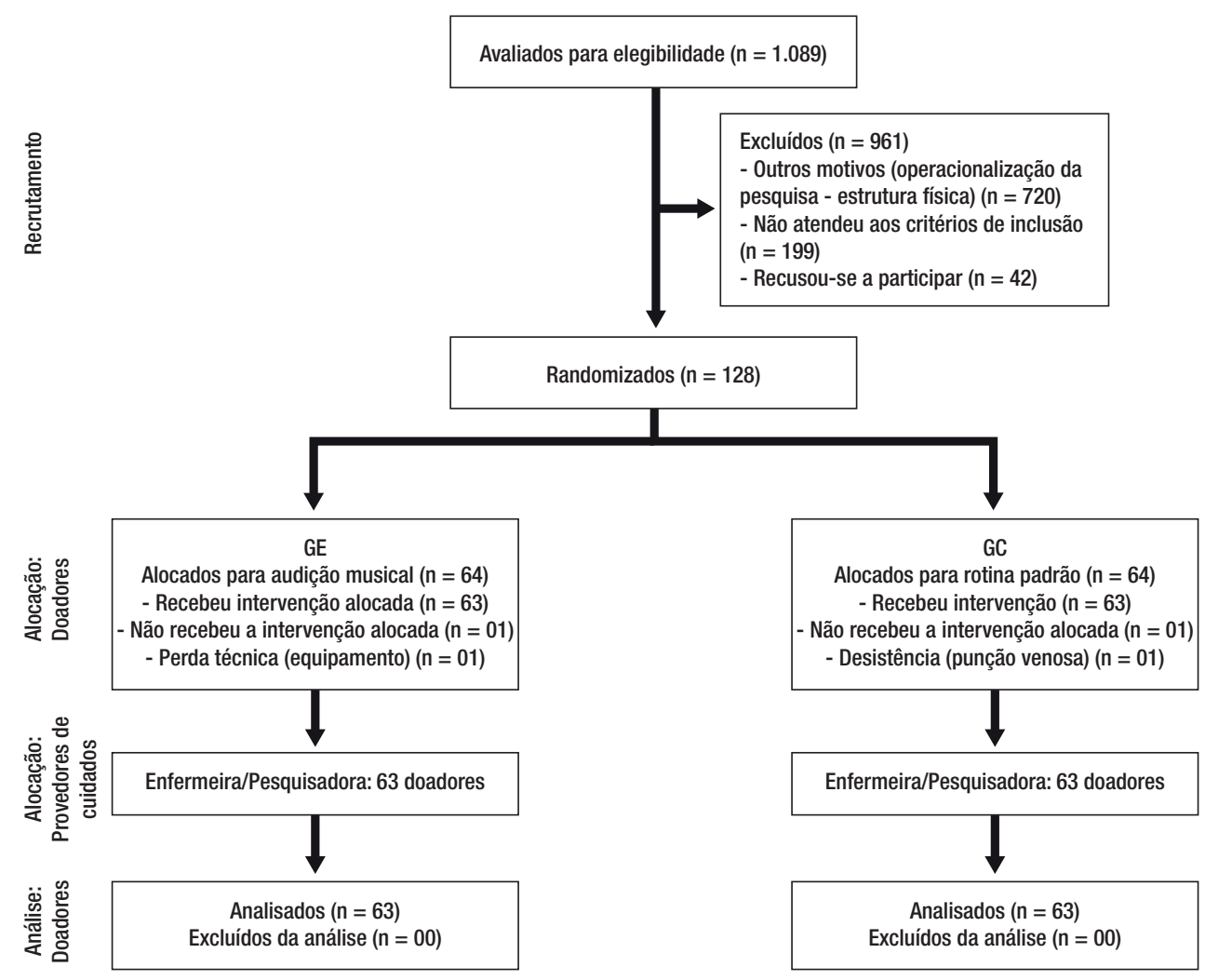

Figura 1. Fluxograma dos participantes envolvidos no estudo, conforme as recomendações do CONSORT Statement 
Para a coleta de dados, elaborou-se um instrumento específico, o qual foi submetido ao processo de validação de conteúdo por três especialistas na questão abordada. Esse instrumento era constituído por questóes relativas à identificação do participante, variáveis sociodemográficas (idade, sexo, nível de escolaridade, estado civil, profissão) e variáveis relacionadas ao processo de doação (turno e tipo de doação, número de doaçôes). Para a avaliação dos níveis de ansiedade empregou-se o Inventário de Ansiedade Traço-Estado (IDATE), um instrumento traduzido e validado para a língua portuguesa, composto por duas escalas elaboradas com a finalidade de mensurar dois conceitos distintos de ansiedade: traço de ansiedade (IDATE-T), isto é, o estado usual de ansiedade do indivíduo ou característica da personalidade, e o estado de ansiedade (IDATE-E), definido como uma condição cognitivo-afetiva transitória, estado de ansiedade atual ou sentimento do indivíduo naquele momento. ${ }^{(18)}$

No presente estudo foram analisados os escores de ansiedade da IDATE-E, na qual as opçóes de resposta são: absolutamente não $(=1)$; um pouco $(=2)$; bastante (=3) e muitíssimo (=4). O escore total varia de 20 a 80 pontos. Após a somatória, para fins de correção, os valores dos itens $1,2,5,8,10,11,15,16,19$ e $20(1=4,2=3,3=2$ e $4=1)$ devem ser invertidos. ${ }^{(19,20)}$

Sobre a mensuração dos parâmetros fisiológicos (pressão arterial, saturação de oxigênio, frequência cardíaca e respiratória), todos foram verificados, antes e após a intervenção ou rotina padrão e, antes da efetivação da doação de sangue. A técnica de avaliação das medidas pressóricas seguiu protocolo recomendado pelo Guidelines for Arterial Hypertension Management in Primary Health Care in Portuguese Language Countries. ${ }^{(21)} \mathrm{Na}$ aferição da frequência cardíaca e saturação de oxigênio, utilizou-se o oxímetro de pulso portátil, adulto, marca More Fitness ${ }^{\circledast}$. Já a frequência respiratória foi mensurada a partir da observação dos movimentos respiratórios (inspiração e expiração) por um minuto.

Os níveis de cortisol sanguíneo foram dosados, antes e após a aplicação da intervenção ou rotina-padrão. As amostras de sangue $(3 \mathrm{a} 5 \mathrm{ml}$ ) foram coletadas por profissionais da equipe de enfermagem e obtidas em dois momentos distintos, sendo a primeira coletada no setor de triagem hematológica, após coleta dos dados de identificação, mensuração dos escores de ansiedade e parâmetros fisiológicos. E a segunda amostra foi obtida na sala de coleta, na poltrona de doação, após avaliação dos escores de ansiedade-estado e mensuração dos parâmetros fisiológicos, antes da doação de sangue.

A coleta de dados ocorreu antes do procedimento de doação de sangue. Os participantes foram selecionados no setor de atendimento ao doador, após aptidão na triagem clínica e hematológica. Foram abordados por uma das pesquisadoras, membro da equipe, sendo informados e convidados a participar do estudo. Para aqueles que atenderam aos critérios de inclusão, foi solicitada a assinatura no Termo de Consentimento Livre Esclarecido (TCLE).

Para os participantes de ambos os grupos, aplicou-se o instrumento de coleta de dados e o IDATE, realizou-se mensuração dos parâmetros fisiológicos e dosagem do cortisol sanguíneo. Os doadores alocados para o GE, foram submetidos à audição musical antes da doação de sangue. A intervenção foi conduzida por uma das pesquisadoras em sala privativa, na qual os doadores receberam o fone de ouvido conectado a um aparelho de MP3 com uma gravação em áudio composta de uma coletânea de músicas instrumentais e eruditas.

O volume foi testado e ajustado previamente para conforto do ouvinte e o ambiente foi preparado com portas fechadas, luzes apagadas e uma posição confortável, conforme recomendam as diretrizes para uso da intervenção musical com finalidade de relaxamento. ${ }^{(13)}$ Os doadores foram orientados a desligarem os aparelhos celulares durante a escuta musical e a permanecerem em posição confortável na poltrona reclinável e com os olhos fechados. $\mathrm{O}$ ambiente foi preparado para a intervenção com diminuição de luminosidade, porta e persianas das janelas fechadas. A temperatura da sala foi mantida em $23^{\circ} \mathrm{C}$, com ajuste do ar-condicionado. A pesquisadora cronometrou o tempo e permaneceu próxima ao doador somente nos primeiros cinco minutos da intervenção, com o intuito de certificar o funcionamento adequado dos equipamentos utilizados, bem como o ajuste satisfatório do volume da música.

O repertório deste estudo constituiu-se de seis músicas instrumentais e eruditas: Cello Suite $n^{0} 1$, 
Prelude (Johann Sebastian Bach); Nocturne Op. 9 n. 2 (Frédéric Chopin); Clarinet Concerto In A Major K622 Adagio (Wolfgang Amadeus Mozart); Gymnopédie n. 1 (Erik Satie); The Carnival of the Animals - Le Cygne (Camille Saint-Saëns) e Piano Concerto n 21, $k 467$, $2^{\circ}$ movimento (Wolfgang Amadeus Mozart).

A duração total da coletânea foi de 26 minutos e 24 segundos. Um mínimo de 20 minutos de tempo de audição ininterrupta é necessário para induzir o relaxamento, acompanhada de uma respiração profunda antes de iniciar a intervenção musical. ${ }^{(13)}$

Após o término da audição musical, o participante era conduzido até a sala de coleta para efetuar sua doação de sangue. Na sala de doação, imediatamente antes da punção venosa, o instrumento para avaliação dos escores de ansiedade-estado foi novamente aplicado, bem como a verificação dos parâmetros fisiológicos. Após a punção venosa para doação de sangue, no momento da coleta das amostras para tipagem sanguínea e sorologias, procedeu-se a coleta de uma nova amostra de sangue para a dosagem do cortisol sanguíneo.

Os participantes alocados no GC não foram submetidos à audição musical antes da doação e seguiram o fluxo habitual até a sala de coleta para efetuar o procedimento de doação de sangue, conforme a rotina padrão preconizada pela Instituição, na qual o candidato à doação após a aptidão nas triagens clínica e hematológica, segue para a sala de coleta para efetivação do procedimento de coleta de sangue. $\mathrm{Na}$ sala de coleta, imediatamente antes da punção venosa, foi novamente aplicado o instrumento para avaliação dos escores de ansiedade-estado, bem como a verificação dos parâmetros fisiológicos e dosagem do cortisol sanguíneo.

O desfecho primário do estudo foi a redução (diferença) dos escores de ansiedade-estado, obtida após a subtração dos escores de ansiedade-estado, pré e pós-intervenção ou rotina padrão, em ambos os grupos. Os desfechos secundários referem-se à redução (diferença) entre os valores de pressão arterial sistólica (PAS), pressão arterial diastólica (PAD), frequência cardíaca $(\mathrm{FC})$, frequência respiratória (FR), saturação de oxigênio (sat. O2) e cortisol sanguíneo. A variável independente constitui-se do repertório de músicas instrumentais e eruditas.

Para análise dos dados empregou-se o software Statistical Package Social Science (SPSS 21.0). A técnica de dupla digitação foi aplicada e o nível de significância utilizado foi $\alpha=0,01$. Para testar a hipótese de homogeneidade dos dois grupos (controle e experimental), foi utilizado o Teste t para amostras independentes nas variáveis quantitativas (idade e número de doaçóes) e o Teste Qui-Quadrado de homogeneidade para variáveis categóricas (sexo e primeira doação). As variáveis contínuas foram submetidas ao teste de normalidade por meio do Teste de Shapiro-Wilk.

Para as variáveis quantitativas empregou-se a estatística descritiva, por meio de medidas descritivas de centralidade e de dispersão. Utilizou-se o Teste $\mathrm{t}$ de Student para amostras independentes, com o propósito de analisar a média da diferença entre os escores de ansiedade-estado pré e pós-intervenção ou rotina padrão entre os grupos e avaliar a eficácia da intervenção sobre a ansiedade-estado (variável desfecho), FC e FR. Empregou-se o Teste não paramétrico de MannWhitney para analisar a média da diferença entre os valores de PAS, PAD, saturação de $\mathrm{O} 2$ e níveis de cortisol, pré e pós-intervenção ou rotina padrão entre grupos, objetivando também avaliar a eficácia da audição musical sobre essas variáveis.

Este estudo atendeu às normas nacionais de pesquisa envolvendo seres humanos e foi submetido aos Comitês de Ética em Pesquisa da Universidade Federal do Triângulo Mineiro, CAAE: 63470216.4.0000.5154, parecer n. 1.916.589 e, da Fundação Hemominas, CAAE: 63470216.4.3001.5118, parecer n. 2.155.174.

\section{Resultados}

A amostra do estudo foi constituída de 126 doadores de sangue, sendo 63 doadores no GE e 63 no GC. Verificam-se, na tabela 1 , os dados relativos à caracterização sociodemográfica, ao processo de doação e os testes de homogeneidade.

Investigou-se a homogeneidade da amostra do estudo quanto ao sexo e às doaçóes efetuadas pela primeira vez (teste Qui-Quadrado) e, idade e número de doaçóes de sangue (Teste t). Os testes evidenciaram que os grupos, controle e experimental foram comparáveis, considerando essas variáveis.

Dos doadores de sangue participantes do estudo, observou-se que a maioria era do sexo femini- 
Tabela 1. Caracterização sociodemográfica, do processo de doação e testes de homogeneidade para as variáveis idade, número de doações de sangue, sexo e primeira doação; considerando os grupos controle e experimental

\begin{tabular}{|c|c|c|c|c|c|}
\hline \multirow{2}{*}{$\begin{array}{l}\text { Variáveis sociodemográficas } \\
\text { e relativas ao processo de } \\
\text { doação }\end{array}$} & \multicolumn{2}{|c|}{$\begin{array}{c}\mathrm{GC} \\
(\mathrm{n}=63)\end{array}$} & \multicolumn{2}{|c|}{$\begin{array}{c}\text { GE } \\
(n=63)\end{array}$} & \multirow[t]{2}{*}{$p$-value } \\
\hline & Média & $\mathrm{DP} \ddagger$ & Média & $\mathrm{DP} \ddagger$ & \\
\hline Idade em anos & 35,13 & 9,22 & 32,49 & 9,34 & $0,114 \|$ \\
\hline \multirow[t]{2}{*}{ Número de doações de sangue } & 6,90 & 11,17 & 6,52 & 9,53 & $0,837 \|$ \\
\hline & \multicolumn{2}{|c|}{$\mathrm{n}(\%)$} & \multicolumn{2}{|c|}{$\mathrm{n}(\%)$} & \\
\hline \multicolumn{6}{|l|}{ Sexo } \\
\hline Feminino & \multicolumn{2}{|c|}{$27(42,9)$} & \multicolumn{2}{|c|}{$37(58,7)$} & \\
\hline Masculino & \multicolumn{2}{|c|}{$36(57,1)$} & \multicolumn{2}{|c|}{$26(41,3)$} & $0,075 \uparrow$ \\
\hline \multicolumn{6}{|l|}{ Nivel de escolaridade } \\
\hline Ensino Fundamental & \multicolumn{2}{|c|}{$11(17,4)$} & \multicolumn{2}{|c|}{$10(15,9)$} & \\
\hline Ensino Médio & \multicolumn{2}{|c|}{$26(41,3)$} & \multicolumn{2}{|c|}{$24(38,1)$} & - \\
\hline Ensino Superior & \multicolumn{2}{|c|}{$26(41,3)$} & \multicolumn{2}{|c|}{$29(46,0)$} & \\
\hline \multicolumn{6}{|l|}{ Estado civil } \\
\hline Casado (a) / união estável & \multicolumn{2}{|c|}{$38(60,3)$} & \multicolumn{2}{|c|}{$37(58,7)$} & \\
\hline Solteiro & \multicolumn{2}{|c|}{$21(33,3)$} & \multicolumn{2}{|c|}{$23(36,5)$} & \\
\hline Divorciado (a) & \multicolumn{2}{|c|}{$04(6,4)$} & \multicolumn{2}{|c|}{$03(4,8)$} & 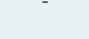 \\
\hline \multicolumn{6}{|l|}{ Tipo de doação } \\
\hline Reposição & \multicolumn{2}{|c|}{ 18(28.6) } & \multicolumn{2}{|c|}{$14(22.2)$} & \\
\hline Espontânea & \multicolumn{2}{|c|}{$38(60.3)$} & \multicolumn{2}{|c|}{$44(69.9)$} & - \\
\hline Convocação & \multicolumn{2}{|c|}{$07(11.1)$} & \multicolumn{2}{|c|}{ 05(7.90) } & \\
\hline \multicolumn{6}{|l|}{ Primeira doação de sangue } \\
\hline Sim & \multicolumn{2}{|c|}{ 14(22.2) } & & & \\
\hline Não & & & & & 1.009 \\
\hline
\end{tabular}

fDP = desvio padrão; $\|$ - teste de homogeneidade (valor de p) referente ao cálculo do teste t; 9 - teste de homogeneidade (valor de p) referente ao cálculo do Teste Qui-Quadrado.

no $(50,8 \%)$, casadas/uniáo estável $(59,5 \%)$, com ensino superior (43,7\%), faixa etária entre 30 e 49 anos (59,5\%), com média de idade de 33,81 anos $(\mathrm{DP}=9,34)$. Em relação ao processo de doação de sangue, os participantes efetuaram suas doaçôes no período matutino $(53,2 \%)$, sendo a maioria, doaçóes do tipo espontânea $(65,10 \%)$. Concernente ao número de doaçóes de sangue, 57,9\% dos doadores já haviam efetuado entre uma e 10 doaçóes, sendo a média de comparecimentos de 6,71 (DP=10,34).

Ao investigar a eficácia da audição musical sobre a média da diferença (redução) entre os escores de ansiedade-estado, parâmetros fisiológicos e nível de cortisol pré e pós-intervenção (análise entre grupos), os resultados da tabela 2 , evidenciaram que a redução dos escores de ansiedade-estado foi maior no grupo submetido à intervenção, sem diferença significativa $(\mathrm{p}=0,31)$. Em relação à pressão arterial sistólica, observou-se que a redução média foi maior no grupo experimental, sem significância estatística $(\mathrm{p}=0,17)$. Quanto à pressão arterial diastólica, a média da diferença foi igual para ambos os grupos, com aumento nos valores médios $(\mathrm{p}=0,98)$.
$\mathrm{Na}$ análise da eficácia da intervenção sobre a frequência cardíaca e frequência respiratória, observou-se redução média maior no grupo experimental com diferença estatisticamente significativa (respectivamente, $\mathrm{p}=0,006 ; \mathrm{p}=0,007)$. Os valores de saturação de $\mathrm{O} 2$ demonstraram que a média foi maior no grupo experimental, contudo sem significância estatística $(\mathrm{p}=0,31)$. Quanto aos níveis de cortisol sanguíneo, os resultados também evidenciaram redução maior no grupo experimental, com diferença significativa entre os grupos $(\mathrm{p}<0,001)$ (Tabela 2$)$.

Tabela 2. Comparação intergrupos da diferença dos escores de ansiedade-estado, níveis dos parâmetros fisiológicos e cortisol sanguíneo, pré e pós-intervenção, considerando os grupos controle e experimental

\begin{tabular}{|c|c|c|c|c|c|c|}
\hline Variáveis/Grupos & & $\mathrm{n}^{*}$ & $\mathrm{M}_{\text {diferenca }} \dagger$ & Mediana & $\mathrm{DP} \ddagger$ & $p$-value§ \\
\hline \multirow{2}{*}{$\begin{array}{l}\text { Ansiedade- } \\
\text { Estado }\end{array}$} & Controle & 63 & $-0,38$ & $-1,00$ & 6.07 & \multirow{2}{*}{$0,31^{\beta}$} \\
\hline & Experimental & 63 & 0,70 & 1,00 & 5,85 & \\
\hline \multirow{2}{*}{$\begin{array}{l}\text { Pressão arterial } \\
\text { (sistólica) }\end{array}$} & Controle & 63 & 0,79 & 0,00 & 9,89 & \multirow{2}{*}{$0,17^{\Omega}$} \\
\hline & Experimental & 63 & 2,68 & 0,00 & 7,90 & \\
\hline \multirow{2}{*}{$\begin{array}{l}\text { Pressão arterial } \\
\text { (diastólica) }\end{array}$} & Controle & 63 & $-1,75$ & 0,00 & 8,71 & \multirow{2}{*}{$0,98^{\Omega}$} \\
\hline & Experimental & 63 & $-1,75$ & 0,00 & 9,25 & \\
\hline \multirow{2}{*}{$\begin{array}{l}\text { Frequência } \\
\text { cardíaca }\end{array}$} & Controle & 63 & 4,73 & 4,00 & 7,01 & \multirow{2}{*}{$0,006^{\beta}$} \\
\hline & Experimental & 63 & 8,70 & 9,00 & 8,74 & \\
\hline \multirow{2}{*}{$\begin{array}{l}\text { Frequência } \\
\text { respiratória }\end{array}$} & Controle & 63 & $-1,40$ & $-2,00$ & 2,64 & \multirow{2}{*}{$0,007^{\beta}$} \\
\hline & Experimental & 63 & 0,03 & 0,00 & 3,15 & \\
\hline \multirow[t]{2}{*}{ Saturação de 02} & Controle & 63 & $-0,21$ & 0,00 & 1,65 & \multirow{2}{*}{$0,31^{\Omega}$} \\
\hline & Experimental & 63 & $-0,35$ & 0,00 & 1,08 & \\
\hline \multirow[t]{2}{*}{ Cortisol } & Controle & 63 & $-1,07$ & $-0,06$ & 4,82 & \multirow{2}{*}{$<0.001^{\Omega}$} \\
\hline & Experimental & 63 & 2,40 & 1,29 & 7,97 & \\
\hline
\end{tabular}

${ }^{*} n=$ número de participantes; $\dagger$ = média da diferença (redução); $\ddagger$ = desvio padrão das diferenças; $\S$ = $p$ - value; $\beta=$ Teste $t$ de Student; $\Omega=$ Teste Mann-Whitney.

\section{Discussão}

Os achados deste estudo evidenciaram que, a audição de um repertório pré-selecionado de músicas instrumentais e eruditas antes da doação de sangue, avaliado por meio do IDATE, não reduziu significativamente os escores de ansiedade-estado. Contudo, evidenciou-se impacto positivo sobre os parâmetros fisiológicos e medidas objetivas, com reduçóes significativas sobre a frequência cardíaca, frequência respiratória e níveis de cortisol sanguíneo.

Tais resultados corroboram outras pesquisas que tiveram como objeto de estudo a utilização da intervenção musical tal como aqui proposta, mas que foram realizadas com pacientes em diferentes contextos ou situaçôes clínicas. Ensaios clínicos realizados com pacientes oncológicos adultos e 
com pacientes portadores de doença renal crônica em hemodiálise, indicaram que o grupo submetido à audição de músicas clássicas, apresentou reduções estatisticamente significativas dos parâmetros fisiológicos avaliados (PA, FR, FC), cortisol e dos níveis de ansiedade-estado. ${ }^{(4,8,22)}$

Reduçóes significativas dos parâmetros vitais, bem como dos escores de ansiedade-estado, foram evidenciadas em outros estudos, seja no contexto da realização de procedimentos invasivos, como endoscopia, ${ }^{(23)}$ aspiração e biópsia de medula óssea, ${ }^{(9)}$ e, no contexto cirúrgico, pré-operatório. ${ }^{(14)}$

Outras pesquisas também confirmaram que a intervenção com músicas relaxantes, além de reduzir os parâmetros fisiológicos, diminuiu os níveis de cortisol. ${ }^{(8,22-25)}$ Especificamente, quanto ao impacto da audição musical sobre os níveis de cortisol, outros estudos também evidenciaram reduções significativas dos níveis deste marcador. ${ }^{(26-28)}$ Para Suzuki et al., ${ }^{(29)}$ a música tem influência direta na redução do cortisol, que é o hormônio do estresse.

Embora as respostas à música sejam frequentemente consideradas subjetivas, as pesquisas sugerem que as variáveis cardiorrespiratórias respondem a estimulação musical de maneira objetiva, ou seja, as respostas subjetivas da música têm evidenciado efeitos físicos bem objetivos, ambos certamente mediados pelo cérebro. ${ }^{(30)}$

As evidências científicas mostram que a música se constitui como terapia complementar propícia para o alívio da ansiedade. Embora algumas pesquisas não indiquem reduçóes significativas dos escores médios de ansiedade-estado, é importante ressaltar a satisfação, cooperação e conforto referidos pelos pacientes frente a utilização dessa ferramenta, antes e durante os procedimentos. ${ }^{(31-33)}$

Salienta-se que um aspecto determinante no uso da intervenção musical com fins ansiolíticos refere-se à escolha do gênero ou estilo musical. Para Krout, ${ }^{(34)}$ a música clássica é utilizada frequentemente para fins de relaxamento, sendo considerado um estilo musical amplo, composto por alguns elementos musicais, que faz com que a música seja percebida como relaxante pelos ouvintes. Idrobo-Ávila et al. ${ }^{(35)} \mathrm{em}$ sua revisão sistemática, destacaram o uso da música clássica nas intervençôes musicais como sendo o estilo musical mais utilizado, executada principalmente em fones de ouvido, com o ouvinte na posição sentada mais frequentemente que na posição supina e com duração das sessốes de audição em torno de 15 a 30 minutos, na maioria dos estudos. Tais achados corroboram com a intervenção proposta na presente pesquisa e reafirmam os resultados encontrados.

Considerou-se como limitaçôes deste estudo, a realização da pesquisa em um único centro; a escolha de um repertório pré-selecionado de músicas instrumentais e eruditas, pois a escolha individualizada da música ainda é um fator controverso na literatura; além das perdas de potenciais participantes, devido à limitação do espaço físico do local do estudo.

\section{Conclusão}

Este estudo permitiu concluir que a audição de um repertório de músicas instrumentais e eruditas pré-selecionadas, foi ineficaz para o controle e redução dos escores de ansiedade-estado. Entretanto, houve redução estatisticamente significativa entre os grupos, em relação aos parâmetros fisiológicos e laboratoriais, como frequência cardíaca, frequência respiratória e níveis de cortisol sanguíneo.

\section{Agradecimentos}

Os autores declaram que parte desta pesquisa recebeu apoio financeiro da Pró-Reitoria de Pesquisa e Graduação (PROPPG) da Universidade Federal do Triângulo Mineiro (UFTM), Edital PROPPG/ UFTM N.02, de 09 de janeiro de 2017, Edital para Fomento a Pesquisadores da UFTM.

\section{Colaborações}

Silva KFN, Felix MMS, Barbosa MH declaram que contribuíram para a concepção do estudo; coleta, análise e interpretação dos dados, redação do artigo, revisão crítica relevante do conteúdo intelectual e aprovação final da versão a ser publicada. Hass VJ, Cruz LF declaram que contribuíram para a concepção do estudo; coleta, análise e interpretação dos dados; aprovação 
final da versão a ser publicada. Barichello E, Pires PS, Mattia ALD declaram que contribuíram com a redação do artigo, revisão crítica relevante do conteúdo intelectual e aprovação final da versão a ser publicada.

\section{Referências}

1. Kılıç SÇ, Doğan E, Sevimligül G, Yücel B, Bolat F, Kavakçı O, et al. Assessing anxiety levels and empathic tendency in blood and platelet donors. Transfus Apheresis Sci. 2013;48(3):297-300.

2. Ditto B, France CR. Vasovagal symptoms mediate the relationship between predonation anxiety and subsequent blood donation in female volunteers. Transfusion. 2006;46(6):1006-10.

3. Cantekin I, Tan M. The influence of music therapy on perceived stressors and anxiety levels of hemodialysis patients. Ren Fail. 2013;35(1):105-9.

4. Firmeza MA, Rodrigues AB, Melo GA, Aguiar MI, Cunha GH, Oliveira PP, et al. Control of anxiety through music in a head and neckoutpatient clinic: a randomized clinical trial. Rev Esc Enferm USP. 2017;51(0):e03201.

5. Galaal K, Bryant A, Deane KH, Al-Khaduri M, Lopes AD. Interventions for reducing anxiety in women undergoing colposcopy. Cochrane Database Syst Rev. 2011 Dec;12(12):CD006013.

6. Li Y, Dong Y. Preoperative music intervention for patients undergoing cesarean delivery. Int J Gynaecol Obstet. 2012;119(1):81-3.

7. Li XM, Zhou KN, Yan H, Wang DL, Zhang YP. Effects of music therapy on anxiety of patients with breast cancer after radical mastectomy: a randomized clinical trial. J Adv Nurs. 2012;68(5):1145-55.

8. Melo GA, Rodrigues AB, Firmeza MA, Grangeiro AS, Oliveira PP, Caetano JA. Musical intervention on anxiety and vital parameters of chronic renal patients: a randomized clinical trial. Rev Lat Am Enfermagem. 2018;26(0):e2978-2978.

9. Shabanloei R, Golchin M, Esfahani A, Dolatkhah R, Rasoulian M. Effects of music therapy on pain and anxiety in patients undergoing bone marrow biopsy and aspiration. AORN J. 2010;91(6):746-51.

10. Nociti JR. Music and anesthesia. Rev Bras Anestesiol. 2010;60(5):455-6.

11. Koelsch S, Jäncke L. Music and the heart. Eur Heart J. 2015;36(44):3043-9.

12. Todres ID. Music is medicine for the heart. J Pediatr (Rio J). 2006;82(3):166-8.

13. Lindquist R, Snyder M, Tracy MF, editors. Complementary \& alternative therapies in nursing. 7th ed. New York: Springer Publishing Company; 2014.

14. Mohammadi A, Ajorpaz NM, Torabi M, Mirsane A, Moradi F. Effects of music listening on preoperative state anxiety and physiological parameters in patients undergoing general surgery: a randomized quasi-experimental trial. Cent Eur J Nurs Midw 2014, 5(4):156-60.

15. Boutron I, Moher D, Altman DG, Schulz KF, Ravaud P. Extending the CONSORT statement to randomized trials of nonpharmacologic treatment: explanation and elaboration. Ann Intern Med. 2008;148(4):295-309.

16. Boutron I, Altman DG, Moher D, Schulz KF, Ravaud P; CONSORT NPT Group. CONSORT Statement for Randomized Trials of Nonpharmacologic Treatments: A 2017 Update and a CONSORT Extension for Nonpharmacologic Trial Abstracts. Ann Intern Med. 2017;167(1):40-7.

17. Dallal GE. Randomization.com. http://www.randomization.com/; 2017. Accessed 05 December 2017.
18. Biaggio AM, Natalício L, Spielberger CD. Desenvolvimento da forma experimental em português do Inventário de Ansiedade Traço-Estado (IDATE), de Spielberger. Arq Bras Psicol. 1977;29(3):31-44. [citado 22 dezembro 2019]. Disponível em: http://bibliotecadigital.fgv.br/ojs/ index.php/abpa/article/viewFile/17827/16571.

19. Spielberger CD, Gorsuch RL, Lushene RE. Inventário de ansiedade traço-estado (IDATE) [Tradução e adaptação de Ângela M. B. Biaggio e Luiz Natalício]. Rio de Janeiro: CEPA; 1979. 60 p.

20. Salles LF, Silva MJ. Efeito das essências florais em indivíduos ansiosos. Acta Paul Enferm. 2012;25(2):238-42.

21. Oliveira GM, Mendes M, Malachias MV, Morais J, Moreira O, Coelho AS, et al. 2017 Guidelines for Arterial Hypertension Management in Primary Health Care in Portuguese Language Countries. Arq Bras Cardiol. 2017;109(5):389-96.

22. Zengin S, Kabul S, Al B, Sarcan E, Doğan M, Yildirim C. Effects of music therapy on pain and anxiety in patients undergoing port catheter placement procedure. Complement Ther Med. 2013;21(6):689-96.

23. Padam A, Sharma N, Sastri OS, Mahajan S, Sharma R, Sharma D. Effect of listening to Vedic chants and Indian classical instrumental music on patients undergoing upper gastrointestinal endoscopy: $A$ randomized control trial. Indian J Psychiatry. 2017;59(2):214-8.

24. Phipps MA, Carroll DL, Tsiantoulas A. Music as a therapeutic intervention on an inpatient neuroscience unit. Complement Ther Clin Pract. 2010;16(3):138-42.

25. Ventura T, Gomes MC, Carreira T. Cortisol and anxiety response to a relaxing intervention on pregnant women awaiting amniocentesis. Psychoneuroendocrinology. 2012;37(1):148-56.

26. Nakayama H, Kikuta F, Takeda H. A pilot study on effectiveness of music therapy in hospice in Japan. J Music Ther. 2009;46(2):160-72.

27. Nilsson $U$. The effect of music intervention in stress response to cardiac surgery in a randomized clinical trial. Heart Lung. 2009;38(3):201-7.

28. Yamamoto M, Naga S, Shimizu J. Positive musical effects on two types of negative stressful conditions. Psychol Music. 2007;35(2):249-75.

29. Suzuki M, Kanamor M, Nagasawa S, Tokiko I, Takayuki S. Music therapyinduced changes in behavioral evaluations, and saliva chromogranin A and immunoglobulin A concentrations in elderly patients with senile dementia. Geriatr Gerontol Int. 2007;7(1):61-71.

30. Bernatzky G, Presch M, Anderson M, Panksepp J. Emotional foundations of music as a non-pharmacological pain management tool in modern medicine. Neurosci Biobehav Rev. 2011;35(9):1989-99.

31. Aba YA, Avci D, Guzel Y, Ozcelik SK, Gurtekin B. Effect of music therapy on the anxiety levels and pregnancy rate of women undergoing in vitro fertilization-embryo transfer: A randomized controlled trial. Appl Nurs Res. 2017;36:19-24.

32. Ko CH, Chen YY, Wu KT, Wang SC, Yang JF, Lin YY, et al. Effect of music on level of anxiety in patients undergoing colonoscopy without sedation. J Chin Med Assoc. 2017;80(3):154-60.

33. Tsivian M, Qi P, Kimura M, Chen VH, Chen SH, Gan TJ, et al. The effect of noise-cancelling headphones or music on pain perception and anxiety in men undergoing transrectal prostate biopsy. Urology. 2012;79(1):32-6.

34. Krout RE. Music listening to facilitate relaxation and promote wellness: integrated aspects of our neurophysiological responses to music. Arts Psychother. 2007;34(2):134-41.

35. Idrobo-Ávila EH, Loaiza-Correa H, van Noorden L, Muñoz-Bolaños FG, Vargas-Cañas R. Different Types of Sounds and Their Relationship With the Electrocardiographic Signals and the Cardiovascular System - Review. Front Physiol. 2018;9(9):525. 